\title{
Disrupting the rhythm of depression: design and protocol of a randomized controlled trial on preventing relapse using brief cognitive therapy with or without antidepressants
}

\author{
Claudi LH Bockting ${ }^{1 *}$, Hermien J Elgersma ${ }^{1,2}$, Gerard D van Rijsbergen ${ }^{1}$, Peter de Jonge ${ }^{3}$, Johan Ormel ${ }^{3}$, \\ Erik Buskens ${ }^{4}$, A Dennis Stant ${ }^{4}$, Peter J de Jong ${ }^{1}$, Frenk PML Peeters ${ }^{5}$, Marcus JH Huibers ${ }^{6}$, Arnoud Arntz ${ }^{6}$, \\ Peter Muris ${ }^{7}$, Willem A Nolen ${ }^{3}$, Aart H Schene ${ }^{8}$, Steven D Hollon ${ }^{9}$
}

\begin{abstract}
Background: Maintenance treatment with antidepressants is the leading strategy to prevent relapse and recurrence in patients with recurrent major depressive disorder (MDD) who have responded to acute treatment with antidepressants (AD). However, in clinical practice most patients (up to 70-80\%) are not willing to take this medication after remission or take too low dosages. Moreover, as patients need to take medication for several years, it may not be the most cost-effective strategy. The best established effective and available alternative is brief cognitive therapy (CT). However, it is unclear whether brief $C T$ while tapering antidepressants (AD) is an effective alternative for long term use of $A D$ in recurrent depression. In addition, it is unclear whether the combination of $A D$ to brief $C T$ is beneficial.

Methods/design: Therefore, we will compare the effectiveness and cost-effectiveness of brief $C T$ while tapering $A D$ to maintenance $A D$ and the combination of $C T$ with maintenance $A D$. In addition, we examine whether the prophylactic effect of $C T$ was due to $C T$ tackling illness related risk factors for recurrence such as residual symptoms or to its efficacy to modify presumed vulnerability factors of recurrence (e.g. rigid explicit and/or implicit dysfunctional attitudes). This is a multicenter RCT comparing the above treatment scenarios. Remitted patients on $A D$ with at least two previous depressive episodes in the past five years $(n=276)$ will be recruited. The primary outcome is time related proportion of depression relapse/recurrence during minimal 15 months using DSM-IV-R criteria as assessed by the Structural Clinical Interview for Depression. Secondary outcome: economic evaluation (using a societal perspective) and number, duration and severity of relapses/recurrences.
\end{abstract}

Discussion: This will be the first trial to investigate whether $C T$ is effective in preventing relapse to depression in recurrent depression while tapering antidepressant treatment compared to antidepressant treatment alone and the combination of both. In addition, we explore explicit and implicit mediators of CT.

Trial registration: Netherlands Trial Register (NTR): NTR1907

\section{Background}

Major depressive disorder (MDD) is projected to rank second on a list of 15 major diseases in terms of burden of disease in 2030 [1]. The major contribution of MDD to disability and health care costs is largely due to its

\footnotetext{
* Correspondence: c.l.h.bockting@rug.nl

'Department of Clinical Psychology, Groningen University, The Netherlands Full list of author information is available at the end of the article
}

highly recurrent nature [2,3]. Accordingly, efforts to reduce the disabling effects of depression should shift to preventing recurrences, especially in patients at high risk of recurrence. Several international guidelines (e.g., $[4,5])$ report that patients remitted from prior depressive episodes belong to such high risk groups. The preventive strategy globally suggested, i.e. continuation of antidepressants (AD) for years, is certainly not in line with
C Biomed Central

() 2011 Bockting et al; licensee BioMed Central Ltd. This is an Open Access article distributed under the terms of the Creative Commons Attribution License (http://creativecommons.org/licenses/by/2.0), which permits unrestricted use, distribution, and reproduction in any medium, provided the original work is properly cited. 
the fact that $70-80 \%$ of the patients are not willing to take AD for a long period of time [6,7]. Many patients prefer psychological interventions instead. Moreover, continuation of AD has clear limitations. First, nonadherence in AD-users is common (50\%, [5-8]). It may also be contraindicated because of somatic illness or side effects. Further, patients' protection from recurrence ceases on discontinuation of AD [9]. Moreover, a recent meta-analysis indicates that with increasing number of previous episodes, patients develop resistance against the prophylactic properties of AD [10]. Finally, the optimal duration of the maintenance phase has not been studied adequately since few studies reported follow-up periods longer than 1 year.

Meta-analyses indicate that cognitive therapy (CT) is not only an effective treatment of MDD, but can also be used as an effective preventive intervention [10-17]. Sequential treatment in which CT is started after remission has proven to be effective in preventing recurrences in patients with recurrent MDD (e.g. for a meta-analysis see [12]). In a previous multicenter RCT enrolling remitted recurrently depressed patients, the efficacy and cost-effectiveness of CT was evaluated added to treatment as usual (TAU) compared with TAU alone [18]. In line with other studies on this preventive CT, CT was effective [6] and cost-effective [Bockting CLH, Dijkgraaf MGW, Hakaart-van Roijen L et al. Costeffectiveness of relapse-prevention cognitive therapy in recurrent depression: a two year study. Submitted] in preventing recurrences over a 2 -year follow-up and also over 5.5 years in patients with multiple previous episodes [19]. In this case TAU included several types of aftercare; e.g. continuation of $\mathrm{AD}$, non-controlled tapering and discontinuation of $\mathrm{AD}$, both in primary and secondary care. Recently, the preventive effects of CT in primary care have been confirmed in patients who have experienced multiple previous episodes [20]. Since in our prior studies we did not plan a controlled tapering of AD treatment versus AD continuation or the combination with $\mathrm{CT}$, we could not examine whether $\mathrm{CT}$ while tapering $\mathrm{AD}$ or combining it with $\mathrm{AD}$ is an effective strategy in preventing relapse in recurrent depression [5,21]. A previous study [22] randomly assigning remitted recurrently depressed patients $(n=40)$ to $C T$ or clinical management, while withdrawing $\mathrm{AD}$, reported a $50 \%$ reduction in relapse rate in CT vs. clinical management over 6-year follow-up (40\% vs. 90\%). This study suggests long term effects of $\mathrm{CT}$ in patients that stopped AD. There is convincing evidence, based on a recent meta-analysis [12], for the preventive effect of $\mathrm{CT}$, and some evidence for specific forms of CT (like Mindfulness Based Cognitive Therapy; MBCT). In the UK a recent study $(\mathrm{n}=123)$ compared CT $(\mathrm{MBCT})$ while tapering $\mathrm{AD}$, against $\mathrm{AD}$ in recurrent depression and revealed relapse rates over 1 year of $47 \%$ for CT while $60 \%$ of the patients relapse in the group that used $\mathrm{AD}$ [23].

\section{Trial objectives and Purpose}

The objective of this study is to examine whether CT is an effective preventive strategy in reducing relapse/ recurrence while tapering $\mathrm{AD}$ compared to continuation of $\mathrm{AD}$ versus the combination of maintenance $\mathrm{AD}$ with CT.

Alongside this effect study an economic evaluation will be performed. In addition, the study explores potential moderators to examine what works for whom. Potential mediators will be examined to explore the working mechanism of preventive CT by assessing implicit and explicit beliefs, coping related factors, symptoms, stressful life events, and their association to risk of relapse to depression, before, between and after treatment and during the follow-up period.

\section{Methods/Design}

In this multi-center randomized controlled three-arm trial with a sample size of 276 participants an 8 session group $\mathrm{CT}$ with guided tapering of $\mathrm{AD}$, will be compared to continuation of maintenance $\mathrm{AD}$ use versus the combination of $\mathrm{CT}$ with maintenance $\mathrm{AD}$ in remitted patients with recurrent MDD. In doing so we stratify on the number of previous episodes and type of aftercare. The effectiveness and cost-effectiveness (societal perspective) of the interventions will be examined with a follow-up of minimal 15 months.

We undertake randomization by telephone to the Psychiatry department of the University Medical Center of Groningen (UMCG). The number of previous depressive episodes and type of care are delineated for stratification reasons.

We monitor the primary outcome (relapse) over a period of minimal 15 months. Assessments by trained assessors who are blind to treatment allocation (and whose blindness is checked within each assessment session) take place directly after the start of the treatment, at three months, nine and 15 months. In between there is additionally a self report assessment at 1.5 months for the research question on mediation. For an overview of the study's procedure see Figure 1.

\section{The interventions \\ CT-arms}

Patients in the two arms including CT (with tapering of $\mathrm{AD}$ and with continuation of $\mathrm{AD}$ ) receive the proposed intervention (8 weekly group sessions). CT helps patients to identify and change presumed vulnerability factors of recurrence, i.e. dysfunctional beliefs, in line with traditional $\mathrm{CT}$ in the acute phase of depression. 
Recruitment by media attention and posters, through recruitment in general practitioners and secondary mental health care centers, i.e. they are asked to refer eligible participants by providing the study's information letter including response form and informed consent

Interested individuals reply to the information letter, or by the study's telephone number or e-mail address (media).

Researchers globally screen participants willing to participate by telephone for inclusion and exclusion criteria (and sends information and a written consent form if participant responded through media)

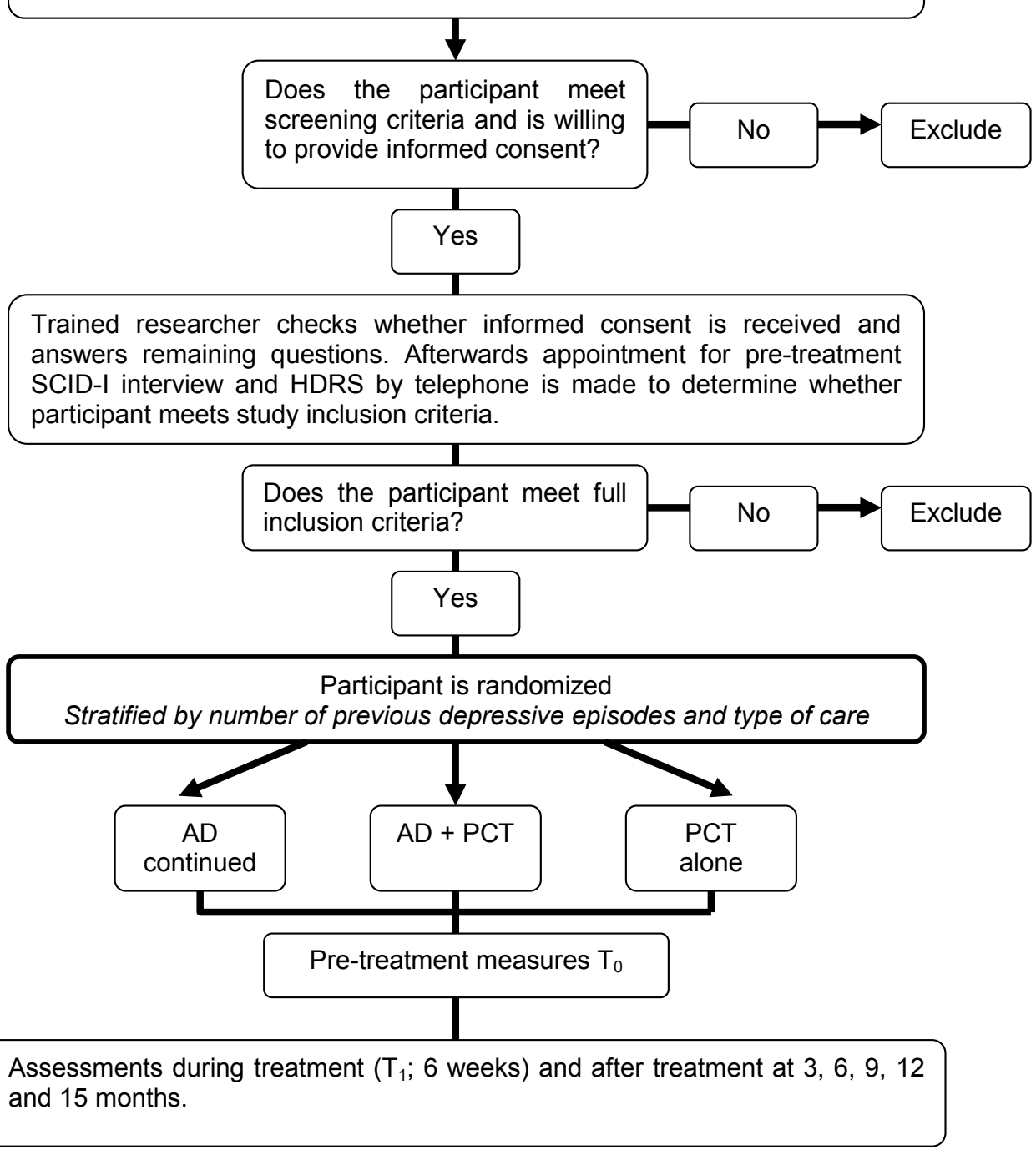

Figure 1 Flow-chart of the study. AD = antidepressant, $\mathrm{PCT}=$ preventive cognitive therapy.

Traditional CT assumes that change of the negative content of thoughts and attitudes are crucial features of successful interventions. More recent cognitive theories of depression, however, emphasize the role of information-processing biases and suggest that these biases are at the core of depression vulnerability and enhanced stress reactivity, via enhancing the elaboration of negative material in memory and reasoning (e.g. [24]). If indeed a difficulty to disengage from negative material is critically involved in the return of complaints, it would 
be important to see whether CT is not only successful in changing content of thoughts and memory associations, but also helps to increase inhibitory control, thereby disrupting the processes of negative emotions and ruminative negative thinking patterns [25].

\section{$A D$-arms}

In the treatment arms where AD will be continued, GP's and psychiatrists will be advised to continue AD prescription at minimal required adequate used dosage ( $\geq 20 \mathrm{mg}$ Fluoxetine equivalent; [6] as recommended by national guidelines [26]. In the treatment arm including guided tapering of AD GP's and psychiatrists will be advised to taper AD in 4 weeks to prevent withdrawal symptoms. In this arm patients will be asked for an intention to taper $\mathrm{AD}$. The patient is allowed to start AD again at any time during the study (this will be monitored).

We will assess compliance and adherence to AD use with the Medication Adherence Scale [27]. We also assess adherence to CT prescription of homework and presence at the sessions. Patients will be encouraged to use medication/do homework as prescribed and doctors/psychiatrists will be encouraged to prescribe therapeutic dosages, as well as discuss problems with adherence frequently.

\section{Sample size}

In total 276 patients will be recruited. This sample size provides $80 \%$ power (alpha 0.05 , two sided) to demonstrate in survival analysis a clinical significant difference of $20 \%$ in relapse/recurrence between AD versus $\mathrm{AD}+\mathrm{CT}$ (relapse $50 \%$ in $\mathrm{AD}$ patients versus $30 \%$ in $\mathrm{AD}+\mathrm{CT}$ ) patients after 15 months, based on a recent meta-analysis on AD versus placebo [10]. Taking into account $15 \%$ attrition, for this comparison $2 \times 98$ participants will be needed. To demonstrate a clinically significant difference of $15 \%$ in relapse/recurrence rate (relapse rate AD: 50\%) between the two treatment arms with continuation of $\mathrm{AD}$ versus the $\mathrm{CT}$ arm while tapering continuation $\mathrm{AD}$ additional 80 participants will be needed.

\section{Inclusion criteria}

We will include patients:

-with at least two previous depressive episodes in the past five years.

-who are currently in remission according to DSM-IV criteria, for longer than 8 weeks and no longer than 2 years.

-that have a current score of $<10$ in the 17 item Hamilton Rating Scale for Depression (HRSD; in line with other prevention studies, e.g. [12,18,28]

-for whom the last episode is at least 2 months and no longer than 2 years ago. -that have been remitted on antidepressant treatment and use AD at entry in the study (delivered in primary or secondary care) for at least 6 months.

\section{Exclusion criteria}

Exclusion criteria are: current mania or hypomania or a history of bipolar illness, any psychotic disorder (current and previous), organic brain damage, alcohol or drug dependency/abuse, predominant anxiety disorder.

\section{Eligibility, informed consent and baseline assessments}

The target population is a high-risk group as identified in several guidelines, (e.g., $[4,5]$ ) that consumes a considerable amount of health care and for whom initial benefits of AD may be lost in the long run. Relapse rates rise with increasing numbers of previous episodes up to $70 \%$ in 5 years [28]. In our previous study, we observed up to $62 \%$ recurrences within 2 years [29].

A highly similar recruitment procedure will be used as in our previous study in which we recruited via media and via referrals from general practitioners or medical specialists in secondary mental health care $[18,19]$. The study will be conducted by a team of clinical psychologists of the department of Psychology at the University of Groningen in collaboration with psychologists of the Rotterdam University and Maastricht University and psychiatrists of the departments of Psychiatry of the University of Amsterdam (AMC) and the Groningen University (UMCG). We collaborate with 16 mental health care sites in The Netherlands. The group intervention will be performed in several cities. Therapists of the sites will be trained with a CT manual to promote treatment integrity.

\section{Informed consent}

We inform patients about the study before they come into the study in two ways. First, by informing the patient through their therapist: if a therapist wants to inform the patient themselves, the patient then receives the information via the therapist and is given a letter containing all the information. If the patient is interested in participating then the participant contacts the researcher. Subsequently, the researcher checks whether the participant understands all aspects of the trial. If they agree to enter the trial, they complete a copy of the consent form and send it back to the researcher.

The second procedure we use is by directly informing the patient in case the patient contacts the researcher (mostly informed by media or by their former therapist/ GP with a letter, by advertisements or interviews). Subsequently, the researcher informs the patient, he/she receives the information in a letter with all the information in it. If the patient is still interested in participating 
after reading the information then the participant contacts the researcher. The researcher checks whether the patient understands all aspects of the trial. If the patient agrees to enter the trial, he/she completes a copy of the consent form and sends it to the researcher.

We remind participants that they can withdraw from the trial at any time and that this will have no consequences for their treatment as usual.

We ask consenting participants to provide information about their socio-demographic background and assess their eligibility in more detail using semi-structured clinical interviews (SCID-I) and self-completed questionnaires. The researchers assess current and past diagnostic status using the Structured Clinical Interview for DSM-IV (SCID, [30]) and the Hamilton Rating Scale for Depression (HRSD, [31]). They ask participants to describe past and current treatments for depression, their attribution of relapse and recurrence and use of AD. If participants meet all inclusion and none of the exclusion criteria for the study, they enter the study.

For the baseline assessment, we ask participants themselves to complete the web based self report questionnaires in two packages: explicit and implicit measures. The first part with assessments starting directly within a week for the AD continuation only arm, and for the other arms briefly before starting preventive CT. The second part of the assessment include implicit measures that will be offered within 2 days after completion of the self report assessments measures. Used self report measures are: the Inventory of Depressive Symptomatology, IDSSR [32], negative life events (Life events questionnaire, LGV [33], self-esteem (Rosenberg's Self-esteem Scale [34]), personality pathology (Personality Diagnostic Questionnaire, PDQ-4 [35]), somatic complaints (LKV [36]), everyday problems (EPCL [37]), hypomania (HCL-32 [38]), direct and indirect costs (TIC-P [39]) and Medication Adherence Questionnaire (MAQ, [27]). a measure of general quality of life (Euro-QOL EQ-5 D [40]), rumination (Ruminative Responses Subscale of the Response Styles Questionnaire, RSQ [41]), dysfunctional attitudes (Dysfunctional Attitudes Scale, DAS [42]) and cognitive reactivity using the LEIDS [43]), acceptance (Acceptance and Action Questionnaire, AAQ [44]) and coping (Utrecht Coping List, UCL [45]). After 6 weeks this set will be repeated with the exception of the TIC-P, LGV, PDQ, MAS and EQ-5 D. During follow-up every three months the following self report assessments will be repeated: IDS-SR, HCL-30, TIC-P, EPCL, and EQ-5 D. For a complete overview of the assessments see table 1.

\section{Outcome measures}

Primary outcome effectiveness: time related proportion of depression relapse/recurrence in a survival analysis
(Cox regression) over a follow-up period of minimal 15 months using DSM-IV-TR criteria as assessed by the Structured Clinical Interview for DSM-IV (SCID, telephonic version, [46]) at 3 months, 9 months and 15 months (current depressive symptomatology and previous 3 and 6 months).

For potential differential (illness related, stress-related and cognitive-related) predictors and mediators the following self report measures will be used at baseline, at 1 and at 3 months (internet based): Inventory of Depressive Symptomatology (IDS-SR; [32]), Negative Life Events Questionnaire [33], Everyday Problem Checklist (EPCL; [37]), Ruminative Responses Subscale of the Response Styles Questionnaire (RSQ; [41]), Dysfunctional Attitude Scale, (DAS-A; [42]), LEIDS [43] and to assess nonadherence to AD with the Medication Adherence Questionnaire (MAQ; [27]). To enable calculating quality adjusted life years required for the economic evaluation, also the EQ5 D also will be administered every 3 months [40]. To test whether CT and/or AD affect implicit attitudes and attentional bias differentially and whether residual difficulty to disengage and residual dysfunctional implicit attitudes are related to the return of depressive symptoms an Implicit Association Test (IAT; [47]) that is also used in the Netherlands Study of Anxiety and Depression http://www.nesda.nl will be used to assess implicit attitudes. A rapid serial visual presentation (RSVP; [48]) task will be used to assess the difficulty to disengage from negative information. Difficulty to disengage will be indexed by the magnitude of the attentional blink when negative self descriptors are presented as the first target and neutral words as the second.

For an overview of the assessments at baseline, in between- and post treatment and follow up assessments see table 1 .

\section{Withdrawal}

Participants can withdraw from treatment or from the study at any time. Nevertheless we ask those who withdraw from the trial treatment $(\mathrm{CT}$ or $\mathrm{CT}+\mathrm{AD}$ or $\mathrm{AD}$ alone) if they are willing to attend all the remaining research appointments or at least to provide minimal data.

\section{Safety monitoring and reporting}

The trial protocol has been approved by an independent medical ethics committee for all included sites (METIGG). Eligible people will be included as participants in the trial only after informed consent has been obtained.

We record and report suspected serious adverse events to the Multi-centre Ethic Committee (METIGG) according to their individual guidelines. 
Table 1 Overview of assessments

\begin{tabular}{|c|c|c|c|c|c|c|c|c|}
\hline Measure & Description & $\mathrm{T}_{0}$ & $\mathrm{~T}_{1}$ & $\mathrm{~T}_{2}$ & $\mathrm{~T}_{3}$ & $\mathrm{~T}_{4}$ & $T_{5}$ & $\mathrm{~T}_{6}^{*}$ \\
\hline IDS-SR & Depressive symptoms & + & + & + & + & + & + & + \\
\hline RSQ & Ruminative responses & + & + & + & & & & + \\
\hline EQ-5D & Quality of life & + & & + & + & + & + & + \\
\hline DAS & Dysfunctional Attitudes & + & + & + & & & & + \\
\hline AAQ & Experiential acceptance and avoidance & + & + & + & & & & + \\
\hline UCL & Coping & + & + & + & & & & + \\
\hline LGV & Life-events & + & & + & & & & + \\
\hline Self-esteem & Self-esteem & + & + & + & & & & + \\
\hline PDQ-4+ & Personality & + & & & & & & \\
\hline LKV & Somatic complaints & + & & & & & & + \\
\hline EPCL & Everyday problem list & + & + & + & + & + & + & + \\
\hline $\mathrm{HCL}-32$ & Hypomania & + & + & + & + & + & + & + \\
\hline TIC-P & Direct/indirect costs & + & & + & + & + & + & + \\
\hline LEIDS & Dysfunctional attitudes & + & + & + & & & & + \\
\hline SCID-I & DSM-IV-TR Axis I disorders & + & & + & & + & & + \\
\hline HDRS & Depressive symptoms and severity & + & & + & & + & & + \\
\hline$\overline{\text { IAT }}$ & Implicit associations & + & & + & & & & \\
\hline RSVP & Ability to disengage from negative information & + & & + & & & & \\
\hline
\end{tabular}

${ }^{*} T 0=$ Baseline, $T 1= \pm 1,5$ month, $T 2=3$ months, $T 3=6$ months, $T 4=9$ months, $T 5=12$ months, T6 = 15 months.

\section{Analysis}

Cox regression analysis (survival analysis) will be performed, including as covariates the stratification variables that will be used in randomization, and 2 additional variables, i.e.: number of previous episodes, type of care (primary/secondary). Analysis will be conducted by intention to treat, including all subjects randomized in the study (including dropouts and patients who did not taper AD), and per protocol, including only subjects satisfying protocol treatment (up to the point in the study where they failed to do so). Statistical significance will be set at $P<.05$. Mixed-model analysis will be used for the other variables, including baseline values of the dependent variable as a covariate in all analyses. We shall use stress measures and implicit and explicit cognitive measures to explore the extent to which they mediate relapse and recurrence during treatment and follow up.

For the economic evaluation the balance between costs and health outcomes will be compared across strategies using a societal perspective. Primary outcome measure: the number of depression-free days. Both short-term and long-term consequences will be compared. Additionally, Quality Adjusted Life Years will be used as outcome.

\section{Discussion}

Recurrent depression is highly prevalent and reducing relapse and recurrence is therefore essential. This trial will be the first comparison of $\mathrm{CT}$ while tapering $\mathrm{AD}$ versus continuation of $\mathrm{AD}$ versus the combination of both. Apart from the evaluation of the effectiveness and cost-effectiveness, we examine what works for whom. The most frequently used preventive strategy, i.e. continuation of AD for years, is not in line with what patients do and want (70-80\% of the patients are not willing to take AD long enough; $[6,7])$. Many patients prefer psychological interventions instead. The results of this study might improve better patient by treatment matching by examining the effects of divers preventive strategies and explore what strategy is best for a person with specific characteristics. In addition, mediation variables will be examined to get more insight into the essential ingredients of the preventive CT used. This might lead the development of more targeted interventions.

In summary, MDD has a highly recurrent nature [2]; accordingly, efforts to reduce the disabling effects of depression should shift to preventing recurrence, especially in patients at high risk of recurrence. The development and evaluation of alternative preventive strategies and their specific working mechanism, apart from AD, are needed to at least disrupt the rhythm of depression for this large patient group. This trial will contribute to improved and more efficient therapeutic regimens to prevent relapse and recurrence in depression.

\section{Appendix 1: Statistical Analysis Plan}

The primary outcome measure will be the time to relapse or recurrence meeting DSM-IV criteria for a major depressive episode (American Psychiatric Association, 1994) on the Structured Clinical Interview for 
DSM-IV (SCID, [46]). Occurrence of relapse or recurrence (current or since the last assessment point) will be assessed after treatment at 3 months, and at nine months and 15 months thereafter by trained psychologists who are blind to treatment condition. The analysis will be by 'intention to treat' (ITT). The time (in weeks) of relapse or recurrence to Major Depression, as defined above, will be the dependent variable in survival analysis. The treatment group and stratification variables will be used as predictors.

For participants who are lost from the trial, available measures will be used and then censored at the time of their last observation. Since only a participant's first relapse or recurrence to Major Depression will contribute to the survival analysis, the subsequent loss of that participant will not affect the analysis. Participants who miss one or more follow-up assessments, but are then assessed at a later time point will be asked about their current and past symptoms according to SCID diagnostic criteria since their last successful assessment. This will enable us to assess the time to relapse and thus to censoring.

In addition, we use Hamilton Rating Scale for Depression interview (HRSD), to assess the severity of depression at all time points. The other quantitative measures used at baseline, before treatment, and every three months are the IDS-Q, HSRS-E, LKV, PDQ-4, DAS, LEIDS, UCL, RRS, AAQ-II, Self-esteem questionnaire, LGV, EPCL, TIC-P and the EQ5-D (used to compute QALY's). We shall calculate the 'area under the curve' (AUC) of each measure to give a single score.

Mixed-model analysis of covariance (ANCOVA) will be used for the quantitative measures. As covariates we shall use the stratification variables (number of episodes and type of aftercare and treatment group, together with treatment adherence either to continuation AD or tapering $\mathrm{AD}$ or to the number of sessions attended). Potential moderators to be examined include gender, number of previous episodes, residual symptoms of depression (e.g. HRSD score and IDS score at baseline) and duration of remission, duration of last episode, familial psychiatric burden, life events (childhood/adult), coping and age of onset.

For mediation analysis, regression will be used to examine pre-post change on $\mathrm{CT}$ versus $\mathrm{AD}$ alone (binary for the dichotomous outcome of relapse and linear regression for the HRSD score, the DAS, LEIDS and daily hassles score (EDPL) during follow-up and the association of this potential change to outcome (relapse/ recurrence) will be explored.

Cost-effectiveness will be evaluated from a societal perspective; costs in and outside the healthcare sector will be part of the analyses. Both short-term and longterm consequences of the studied interventions will be taken into account. For the short term analysis, a time horizon of 15 months will be applied, during which information on costs and health outcomes will be prospectively collected using the TIC-P. The primary outcome measure of the cost-effectiveness analysis is the number of depression-free days as assessed by the SCID. In the additionally planned cost-utility analysis, QALY's (Quality Adjusted Life Years) will be used as the primary outcome measure as assessed by the EQ5-D. Medical costs that will be assessed include costs related to CT, medication use, hospital admissions, and contacts with healthcare professionals. Outside the healthcare sector, various costs of informal care and productivity losses will be included. Unit prices will largely be based on Dutch standard prices [49]. Costs and health outcomes will be discounted in accordance with Dutch guidelines. The bootstrap method will be applied to estimate nonparametric confidence intervals for mean differences in costs between groups. Furthermore, cost-effectiveness acceptability curves will be used to inform decisionmakers on the probability that the studied intervention is cost-effective. The long-term consequences of CT versus AD will be addressed by a decision type model, focusing on a period of 20 years. Main aspects of the model will be based on data collected in the prospective part of the trial, literature on the patient population under study, and expert opinions. Important cost types that are expected to demonstrate differences between the two treatment arms over a time frame of 20 years include costs related to AD use, contacts with healthcare professionals, and lost productivity. Also, scenario analyses reflecting the duration of effect will be conducted to evaluate the period of sustained effect which results in a break even between costs and long term savings. The primary health outcome applied in the long-term model will be the QALY. Since QALY's cannot directly be assessed during the 20 years time horizon of the long-term analysis, previously published data on longterm QALY outcomes in comparable patient populations will be used when available (or assumptions will have to be made). Sensitivity analyses will focus on variations of applied probabilities, QALY estimates, and influential cost components.

\section{Acknowledgements}

The research is funded by ZONMW: The Netherlands association for Health research and Development (171002401) to CLH Bockting Associate professor of Clinical Psychology and by ZONMW, The Netherlands association for Health research and Development, OOG Grant (100002035) to HJ Elgersma and CLH Bockting. The Netherlands Organization for Scientific Research (NWO) funded this manuscript.

\section{Author details}

${ }^{1}$ Department of Clinical Psychology, Groningen University, The Netherlands. ${ }^{2}$ Mental health care center Accare, The Netherlands. ${ }^{3}$ Department of Psychiatry, University Medical Center Groningen, Groningen University, The 
Netherlands. ${ }^{4}$ Medical Technology Assessment, Department of Epidemiology, University Medical Center Groningen, Groningen University, The Netherlands. ${ }^{5}$ Department of Psychiatry and Neuropsychology, Maastricht University Medical Center, The Netherlands. ${ }^{6}$ Department of Clinical Psychological Science, University of Maastricht, The Netherlands. ${ }^{7}$ Department of Clinical and Health Psychology, Erasmus University, Rotterdam, The Netherlands. ${ }^{8}$ Department of Psychiatry, Academic Medical Center, University of Amsterdam, Amsterdam, The Netherlands. ${ }^{9}$ Vanderbilt University, Department of Psychology, Nashville, Tennessee, USA.

\section{Authors' contributions}

CB drafted this paper (which was added to and modified by all other authors) and wrote the treatment manual for the used intervention, WN wrote the protocol for tapering $A D$, all authors (except GvR) contributed to the design of the study and CB and PdJe to the analytic strategy. All authors read and approved the final manuscript.

\section{Competing interests}

CB participated in a discussion on treatment for depression for a web-based course of Wyeth once on 1/11/2007. FP received speakers' fees from GlaxoSmithKline, Wyeth, Astra Zeneca, Lundbeck, Eli Lilly, Servier, and Janssen-Cilag. WN received grants from Netherlands Organisation for Health Research and Development, Stanley Medical Research Institute, Astra Zeneca, Eli Lilly, GlaxoSmithKline and Wyeth, he received speaker's fees from Astra Zeneca, Eli Lilly, Pfizer, Servier, Wyeth and was in the advisory board of Astra Zeneca, Cyberonics, Eli Lilly, GlaxoSmithKline, Pfizer and Servier. All other authors declare that they have no competing interests.

Received: 10 December 2010 Accepted: 12 January 2011 Published: 12 January 2011

\section{References}

1. Mathers $C D$, Loncar D: Projections of global mortality and burden of disease from 2002 to 2030. PLoS Med 2006, 3:442.

2. Murray CJ, Lopez AD: Regional patterns of disability-free life expectancy and disability-adjusted life expectancy: global Burden of Disease Study. Lancet 1997, 10(349):1347-52.

3. Ormel J, Petukhova M, Chatterji S, Aguilar-Gaxiola S, Alonso J, Angermeyer MC, Bromet EJ, Burger H, Demyttenaere K, de Girolamo G, Haro JM, Hwang I, Karam E, Kawakami N, Lépine JP, Medina-Mora ME, Posada-Villa J, Sampson N, Scott K, Ustün TB, Von Korff M, Williams DR, Zhang M, Kessler RC: Disability and treatment of specific mental and physical disorders across the world: Results from the WHO World Mental Health Surveys. Br J Psychiatry 2008, 192:368-375.

4. American Psychiatric Association: Practice guidelines for the treatment of psychiatric disorders: Compendium 2008 Washington, D.C; 2008.

5. Clinical NICE Depression: Management of depression in primary and secondary care The National Collaborating Centre for Mental Health; 2009.

6. Bockting CLH, ten Doesschate MC, Spijker J, Spinhoven Ph, Schene AH, the DELTA study group: Continuation and maintenance use of antidepressants in recurrent depression. Psychoth Psychosom 2008, 77:17-26.

7. Meijer WE, Heerdink ER, Leufkens HG, Herings RM, Egberts AC, Nolen WA: Incidence and determinants of long-term use of antidepressants. European J Clin Pharmacology 2004, 60:57-61.

8. ten Doesschate MC, Bockting CLH, Schene AH: Adherence to continuation and maintenance antidepressant use in recurrent depression. J Clin Psychiatry 2009, 70:63-9.

9. Viguera AC, Baldessarini RJ, Friedberg J: Discontinuing antidepressant treatment in major depression. Harv Rev Psychiatry 1998, 5:293-306

10. Kaymaz N, van Os J, Loonen AJ, Nolen WA: Evidence that patients with single versus recurrent depressive episodes are differentially sensitive to treatment discontinuation: a meta-analysis of placebo-controlled randomized trials. J Clin Psychiatry 2008, 69:1423-36.

11. Beck AT: The current state of cognitive therapy: a 40-year retrospective. Arch Gen Psychiatry 2005, 62:953-9.

12. Vittengl JR, Clark LA, Dunn TW, Jarrett RB: Reducing relapse and recurrence in unipolar depression: a comparative meta-analysis of cognitive-behavioral therapy's effects. J Consult Clin Psychol 2007, 75:475-88.
13. DeRubeis RJ, Hollon SD, Amsterdam JD, Shelton RC, Young PR, Salomon RM, O'Reardon JP, Lovett ML, Gladis MM, Brown LL, Gallop R: Cognitive Therapy versus Medications in the Treatment of Moderate to Severe Depression. Arch Gen Psychiatry 2005, 62:409-16.

14. Hollon SD, DeRubeis RJ, Shelton RC, Amsterdam JD, Salomon RM, O'Reardon JP, Lovett ML, Young PR, Haman KL, Freeman BB, Gallop R: Prevention of relapse following cognitive therapy vs medications in moderate to severe depression. Arch Gen Psychiatry 2005, 62:417-27.

15. Hollon SD, Stewart MO, Strunk D: Enduring effects for cognitive behavior therapy in the treatment of depression and anxiety. Annu Rev Psychol 2006, 57:285-315.

16. Clarke GN, Hornbrook M, Lynch F, Polen M, Gale J, Beardslee W, $\mathrm{O}^{\prime}$ Connor E, Seeley J: A randomized trial of a group cognitive intervention for preventing depression in adolescent offspring of depressed parents. Archives Gen Psychiatry 2001, 58:1127-34.

17. Dobson KS, Hollon SD, Dimidijan S, Schmaling KB, Kohlenberg RJ, Gallop R, Rizvi SL, Gollan JK, Dunner DL, Jacobson NS: Randomized trial of behavioral activation, cognitive therapy, and Antidepressant medication in prevention of relapse and recurrence in major depression. J Cons Clin Psychol 2008, 76:468-77.

18. Bockting CLH, Schene AH, Spinhoven Ph, Koeter MWJ, Wouters LF, Huyser J, Kamphuis $\mathrm{JH}$ : Preventing recurrence in recurrent depression using cognitive therapy. J Cons Clin Psychol 2005, 73:647-657.

19. Bockting CLH, Spinhoven Ph, Koeter MWJ, Schene AH: Long term effects of preventive cognitive therapy in recurrent depression using: 5.5 years follow-up. J Clin Psychiatry 2009, 70:1621-8.

20. Conradi HJ, de Jonge P, Ormel J: Differential treatment response in depressed patients with multiple prior episodes: CT versus the GP; Cognitive-behavioural therapy v. usual care in recurrent depression. BrJ Psychiatry 2008, 193:505-6.

21. Bockting CLH: The rhythm of depression: The course of recurrent depression and prevention of relapse using cognitive therapy. PhD thesis Amsterdam; 2006.

22. Fava GA, Ruini C, Rafanelli C, Finos L, Conti S, Grandi S: Six-year outcome of cognitive behavior therapy for prevention of recurrent depression. Am J Psychiatry 2004, 161:1872-1876.

23. Kuyken W, Byford S, Taylor RS, Watkins E, Holden E, White K, Barrett B, Byng R, Evans A, Mullan E, Teasdale JD: Mindfulness-based cognitive therapy to prevent relapse in recurrent depression. J Consult Clin Psychol 2008, 76:966-978.

24. de Jong PJ, Pasman W, Kindt M, van den Hout MA: A reaction time paradigm to assess (implicit) complaint-specific dysfunctional beliefs. Behaviour Res Therapy 2001, 39:101-113.

25. Beevers CG: Cognitive vulnerability to depression: A dual process model. Clin Psychol Review 2005, 25:975-1002.

26. Multidisciplinary Guidelines (Dutch). Guidelines Depression and Anxiety 2009

27. Morisky DE, Green LW, Levine DM: Concurrent and predictive validity of a selfreported measure of medication adherence. Med Care 1986, 24:67-74.

28. Ma SH, Teasdale JD: Mindfulness-based cognitive therapy for depression: Replication and exploration of differential relapse prevention effects. $J$ Cons Clin Psychol 2004, 72:31-40.

29. Bockting CLH, Spinhoven Ph, Koeter MW, Wouters LF, Schene AH, the DELTA study group: Prediction of recurrence in recurrent depression and the influence of consecutive episodes on vulnerability: a 2-year prospective study. J Clin Psychiatry 2006, 67(5):747-755.

30. Spitzer RL, Williams JBW, Gibbon M, First MB: The Structured Clinical Interview for DSM-III-R (SCID 1) history, rationale, and description. Arch Gen Psychiatry 1992, 49(8):624-629.

31. Hamilton M: A rating scale for depression. J Neurol Neurosurg Psychiatry 1960, 23:56-62.

32. Rush AJ, Gullion CM, Basco MR, Jarrett RB, Trivedi MH: The Inventory of Depressive Symptomatology (IDS): psychometric properties. Psychol Med 1996, 26:477-86.

33. Kraaij $V$, De Wilde EJ: Negative life events and depressive symptoms in the elderly: A life span perspective. Aging and Mental Health 2001, 5:84-91.

34. Franck E, De Raedt R, Barbez C, Rosseel Y: Psychometric properties of the Dutch Rosenberg self-esteem scale. Pscyhologica Belgica 2008, 48(1):25-35.

35. Akkerhuis GW, Kupka RW, van Groenestijn MAC, Nolen WA: PDQ-4+ Vragenlijst voor persoonlijkheidskenmerken Lisse: Swets \& Zeitlinger; 1996. 
36. van Hemert AM: Lichamelijke klachten vragenlijst Leids Universitair Medisch Centrum; 2003.

37. Vingerhoets AJJM, van Tilburg MAL: Everyday Problem Checklist (EPCL) Lisse: Swets \& Zeitlinger B.V; 1994.

38. Angst J, Adolfsson R, Benazzi F, Gamma A, Hantouche E, Meyer TD, Skeppar P, Vieta E, Scott J: Towards the self-assessment tool for hypomanic symptoms in outpatients. J Affective Disorders 2005, 88:217-233.

39. Hakkaart-van Roijen L, van Straten A, Donker M, Tiemens B: Handleiding Trimbos/iMTA questionnaire for Costs associated with Psychiatric illness (TiC-P) Instituut voor Medische Technology Assessment, Erasmus Universiteit Rotterdam; 2002

40. EuroQol Group: EuroQol: a new facility for the measurement of healthrelated quality of life. Health Policy 1990, 16:199-208.

41. Treynor W, Gonzalez R, Nolen-Hoeksema S: Rumination reconsidered: A psychometric analysis. Cogn Therapy Res 2003, 27:247-259.

42. Weissman AN: The Dysfunctional Attitude Scale. Academic dissertation University of Pennsylvania (Dissertation Abstracts International: 40, 1389B1390B); 1979.

43. Van der Does W: Cognitive reactivity to sad mood: structure and validity of a new measure. Behav Res Ther 2002, 40:105-20.

44. Hayes SC, Strosahl KD, Wilson KG, Bissett RT, Pistorello J, Toarmino D, Polusny MA, Dykstra TA, Batten SV, Bergan J, Stewart SH, Zvolensky MJ, Eifert GH, Bond FW, Forsyth JP, Karekla M, Curry SM: Measuring Experiential Avoidance: A preliminary test of a working model. Psychol Record 2004, 54(4):553-578.

45. Schreurs PJG, Willige van den G, Brosschot JF, Tellegen B, Graus GMH: Herziene handleiding De Utrechtse Coping Lijst: UCL. Omgaan met problemen en gebeurtenissen Lisse: Swes \& Zeitlinger; 1993.

46. First MB, Gibbon M, Spitzer RL, Williams JBW: User Guide for the Structured Clinical Interview for DSM-IV Axis 1 Disorders. Washington, DC: American Psychiatric Association; 1996.

47. Greenwald AG, McGhee DE, Schwartz JLK: Measuring individual differences in implicit cognition. The Implicit Association Test. J Personality Social Psychol 1998, 74:1464-1480.

48. Koster EHW, De Raedt R, Verschuere B, Tibboel H, de Jong PJ: Enhanced emotioninduced attentional blink for negative information in dysphoria. Depression and Anxiety 2009, 26:16-22.

49. Oostenbrink JB, Koopmanschap MA, Rutten FFH: Manual for cost studies, methods and standard prices for economic evaluation in health care [In Dutch: Handleiding voor kostenonderzoek, methoden en richtlijnprijzen voor economische evaluaties in de gezondheidszorg. Geactualiseerde versie] Amstelveen: Health Care Insurance Council; 2004.

\section{Pre-publication history}

The pre-publication history for this paper can be accessed here: http://www.biomedcentral.com/1471-244X/11/8/prepub

doi:10.1186/1471-244X-11-8

Cite this article as: Bockting et al:: Disrupting the rhythm of depression: design and protocol of a randomized controlled trial on preventing relapse using brief cognitive therapy with or without antidepressants. BMC Psychiatry 2011 11:8.

\section{Submit your next manuscript to BioMed Central and take full advantage of:}

- Convenient online submission

- Thorough peer review

- No space constraints or color figure charges

- Immediate publication on acceptance

- Inclusion in PubMed, CAS, Scopus and Google Scholar

- Research which is freely available for redistribution

Submit your manuscript at www.biomedcentral.com/submit
Biomed Central 